\title{
The Influence of Psychological Acceptance and Internalizing in University Students upon Smartphone Addiction: The moderated Mediating Effect of Adverse Childhood Experiences
}

\author{
Junghye Sung ${ }^{1}$, Minkyu Cho ${ }^{2}$ and Choonkyung Kim ${ }^{3 *}$ \\ Department. of Child and Family, Kyungpook National Univ, South Korea \\ ${ }^{1}$ sjhye@knu.ac.kr, ${ }^{2}$ familystark@knu.ac.kr, ${ }^{3 *} k c k y u n g @ k n u . a c . k r$
}

\begin{abstract}
The purpose of this study was to verify the moderated mediating effect of adverse childhood experiences in the influence of university students' psychological acceptance upon the smartphone addiction tendency mediated by the internalizing problems. To conduct this study, a questionnaire survey was carried out targeting students who are attending universities located in Daegu and Gyeongbuk after obtaining the approval from IRB. The survey period ran from October 5, 2019 to December 10, 2019. As a result, of distributing 570 self-report questionnaires, a total of 546 subjects were analyzed, excluding 11 university students who disagree and 13 university students who did not respond to questions. The research analysis was carried out by using SPSS 18.0 and PROCESS macro. The results of this study are as follows. The moderated mediating effect of adverse childhood experiences was significant in the influence of university students' psychological acceptance upon smartphone addiction tendency mediated by the internalizing problems. It was indicated that the higher adverse childhood experience level leads to the less conditional indirect effect. These findings are suggesting the necessity of the preventive approach for reducing smartphone addiction tendency in undergraduates, of the development in a counseling program, and of the continuous research related to this.
\end{abstract}

Keywords: Psychological acceptance, Internalizing problems, Smartphone addiction tendency, Adverse childhood experiences, Moderated mediation effect

\section{Introduction}

As a smartphone is universalized and comes to be more diverse in function due to the rapid development in IT technology, the smartphone is proceeding with expanding its influence upon the life of modern people around the world. Especially, as a result of what the Pew Research as a U.S. market research company surveyed the percentage of smartphone usage targeting 27 countries in the world, our country's smartphone penetration rate appeared to be $95 \%$. The smartphone distribution rate was indicated to be the highest among the surveyed nations [1]. The smartphone based on high speed and portability not only offered new environment and increased social communication using the collaborative participation and the collective intelligence but also provided an opportunity available for enjoying entertainment or leisure through various applications. Thanks to convenience and accessibility in this way, the smartphone became the existence indispensable to us. However, similarly like a coin of having two sides, there are both upside and downside together even in the use of smartphone. The

Article history:

Received (January 8, 2021), Review Result (February 12, 2021), Accepted (March 23, 2021) 
excessive use of smartphone may bring about addiction-related problems as well as physical discomforts such as turtle neck, amblyopia and sleep disorder.

As the smartphone addiction is the condition of being unable to control smartphone usage on its own, it implies what the excessive use of smartphone leads to the occurrence in the dangers of everyday life or in the conflicts with own neighbors and in the health problem [2]. Particularly in case of university students, they are in the period of corresponding to early adulthood of leading to becoming psychologically independent from their parents, thereby coming to be recognized their autonomy by their parents, resulting in coming to be freer to use smartphone than adolescence and in being more likely to be immersed in smartphone. Accordingly, the approach is needed for encouraging the sound use of smartphone and for reducing the side effects of pathological use.

People who have a problem related to addiction mostly have irrational beliefs or ideas, thereby showing vulnerability psychologically. Owing to this, the cognitive behavioral therapy was previously emphasized with the focus on a change in problematic thinking and in negative emotion or thought as the psychological intervention technique in order to solve an addictionrelated problem. On the other hand, rather than directly attempting a change in cognitive contents, an interest is recently rising in ACT (Acceptance and Commitment Therapy) of helping it escape its influence through making it experience that a thought or emotion is just an idea and feeling, but is not what it really is, with accepting the cognitive process itself that is difficult to be changed. In particular, as the psychological acceptance implies what experiences and accepts as it is without avoiding the present moment, it leads to moving in a constructive direction so that the behavior can be helpful for oneself with regarding all experiences as valuable [3]. The psychological acceptance is on a continuous line that is linked to a high level until willingly experiencing from a low level like enduring. The low psychological acceptance level leads to the insufficient ability of possibly tolerating own emotion or thinking and to evading this, resulting in coming to experience the difficulty in emotional regulation and further to suffer the internalizing problems like depression, anxiety or contraction. This can be known the necessity of inquiring into the internalizing problem as a factor of being affected by psychological acceptance based on the finding by Masuda et al., [4] as saying that the psychological acceptance may be effective for lowering the negative emotional expression.

This internalizing problem can function not only as the outcome variable of psychological acceptance but also as the reason variable of smartphone addiction tendency. As the internalizing problem is a part related to the passive and socially daunted behavior and to the negative emotion like depression or anxiety, it leads to experiencing a difficulty in managing daily life with being adjusted to reality. Examining the aspect in relation to social withdrawal that is one of the internalizing problems, the case of having difficulty in forming relationship and exchanging with other people amidst society to which oneself belongs leads to inordinately using the online space in order to meet a desire for the unsatisfied relationship in reality while coming to be isolated socially. Also, through the finding [5] as saying that the internalized problem such as depression, anxiety or social withdrawal becomes a factor of making it susceptible to the smartphone addiction, what decreases the internalizing problem like depression or social withdrawal can be known to be able to function as a factor available for diminishing the smartphone addiction tendency.

Meanwhile, according to the attachment and trauma theory, it was mentioned that the problematic behavior of being shown in adulthood is associated with the negative experience of having been undergone within family relations during childhood, and that the adverse childhood experience made it difficult for healthy psychological growth with going through the developmental stage after this and made it apt to show negative image in diverse aspects such 
as the dysfunctional emotional regulation strategy or the absorption in an addictive behavior [6]. In the researches by Lee and Ahn [7], it was claimed that the adverse childhood experience comes to be left aftereffect like depression, anxiety or social withdrawal with becoming adulthood after this. In the researches by Gang and Kim [8], the research outcome was shown as saying that the more experience in child abuse from parents leads to the higher smartphone addiction level. In other words, it may be varied the influence upon the psychological acceptance, the internalizing problem and the smartphone addiction tendency in university students depending on the adverse childhood experience level.

Overall, it can be predicted that the psychological acceptance in undergraduates may have influence upon smartphone addiction tendency mediated by internalizing problems, and that the adverse childhood experiences may function as a moderating variable in the process that the psychological acceptance affects internalizing problems. Hence, this study is aimed to inspect a model with the combination of a mediation model and a moderation model based on the model of verifying a moderated mediating effect. This study established the research problems as follows.

Research Problem: what about a moderated mediating effect of the adverse childhood experiences in the influence of undergraduates' psychological acceptance upon smartphone addiction tendency through internalizing problems?

\section{Research method}

\subsection{Research Subjects}

To conduct this study, a questionnaire survey was carried out targeting students who are attending universities located in Daegu and Gyeongbuk after obtaining the approval from IRB (Approval Number: KNU2019-0107). The survey period ran from October 5, 2019 to December 10, 2019. As a result, of distributing 570 self-report questionnaires, a total of 546 subjects were analyzed, excluding 11 university students who disagree and 13 university students who did not respond to questions.

\subsection{Measurement tool}

\subsubsection{Psychological acceptance}

To gauge psychological acceptance, the Korean Acceptance Action Questionnaire-II was used that Heo, Choe and Jin [9] adapted the Acceptance Action Questionnaire developed by Hayes et al., [10]. This scale is a single factor and is composed of totally 8 items. As the response mode to each question is based on 7-point Likert scale, it implies that the higher score mean leads to the higher psychological acceptance level. As a result of analyzing reliability in order to look into the item internal consistency, Cronbach's $\alpha$ coefficient appeared to be .853 .

\subsubsection{Internalizing problems}

To measure internalizing problems, the NEO-II Personality Test was applied that was developed by Cosata and McCrae [11] and was standardized by Ahn and Ahn [12]. This study was checked focusing on social withdrawal, faintheartedness and emotional shock among subfactors of neurosis according to the research objective. In the research analysis, the standardized $\mathrm{T}$ score was used. It signifies that the higher average value leads to the higher internalizing problems. 


\subsubsection{Smartphone addiction tendency}

To survey smartphone addiction tendency, SAS-Bwas used that Lee et al., [13] developed. Sub-factors of this scale comprise salience, conflict, mood regulation, tolerance, withdrawal and recurrence and are composed of totally 24 items by 4 questions, respectively. As the response mode to each question is based on 5-point Likert scale, it means that the higher score leads to the higher smartphone addiction tendency. In consequence of parsing reliability of this scale, Cronbach's $\alpha$ coefficient was indicated to be .923 in the whole, .755 in salience, .722 in conflict, .766 in mood regulation, .773 in tolerance, .784 in withdrawal and .727 in recurrence.

\subsubsection{Adverse childhood experiences}

To gauge adverse childhood experiences, ACE scale was used that was developed by Centers for Kisease Control and Prevention based on the Conflict Tactics Scale by Straus [14] and on the Childhood Trauma Questionnaire by Bernstein et al., [15]. It is what checked the adverse effects of a family during childhood with growing, it consists of totally 10 items. It implies that the higher total score leads to the higher adverse childhood experience level.

\subsection{Data analysis}

This study was aimed to verify the moderated mediating effect of adverse childhood experiences in the influence of university students' psychological acceptance upon smartphone addiction tendency through internalizing problems. Statistical significance was inspected at the significance level of .05. The specific analytical procedures are as follows. First, to validate reliability of each variable, the item internal consistency coefficient (Cronbach's $\alpha$ ) was calculated. Second, to examine correlation between variables, Pearson's correlation analysis was carried out. Third, to prove the moderated mediating effect, PROCESS macro was applied. In addition, to look into a size of a mediating effect that is varied depending on a moderating variable, the statistical significance of a conditional indirect effect was inspected with a bootstrapping method.

\section{Results}

\subsection{Correlation among major variables}

As a result of presuming the skewness and kurtosis of the undergraduates' psychological acceptance, internalizing problems, smartphone addiction tendency, and adverse childhood experiences ahead of examining a correlation among main variables, it was shown that the absolute value of standard skewness index is under 3 and that the absolute value of standard kurtosis index is less than 10 in terms of a measurement variable. Thus, the skewness and kurtosis of measurement variables in this study can be seen to satisfy the normal distribution assumption [16]. As a result of figuring out a correlation among undergraduates' psychological acceptance, internalizing problems, smartphone addiction tendency and adverse childhood experiences based on this, the significant correlation among all variables was shown. Examining concretely, the psychological acceptance reflected a negative correlation with internalizing problems $(\mathrm{r}=-.612, \mathrm{p}<.05)$, smartphone addiction tendency $(\mathrm{r}=-.409, \mathrm{p}<.05)$ and adverse childhood experiences $(\mathrm{r}=-.113, \mathrm{p}<.05)$. The internalizing problems showed a positive correlation with smartphone addiction tendency $(\mathrm{r}=.522, \mathrm{p}<.05)$ and adverse childhood experiences $(\mathrm{r}=.173, \mathrm{p}<.05)$. The smartphone addiction tendency appeared to express a positive correlation with adverse childhood experiences $(r=.239, \mathrm{p}<.05)$. 
Table 1. Correlation among major variables

\begin{tabular}{|c|c|c|c|c|}
\hline Variable & 1 & 2 & 3 & 4 \\
\hline 1 & 1 & & & \\
\hline 2 & -.612 & 1 & & \\
\hline 3 & -.409 & .522 & .239 & 1 \\
\hline 4 & -.113 & .173 & -.088 & 1.275 \\
\hline Kurtosis & .745 & .099 & .079 & 1.602 \\
\hline Skewness & -.657 & .132 & & \\
\hline
\end{tabular}

\subsection{A moderated mediating effect of the adverse childhood experiences}

To prove whether a mediating effect that the psychological acceptance influences the smartphone addiction tendency after passing through the internalizing problems is varied depending on the adverse childhood experience level as a moderating variable, it used model No. 7 of PROCESS macro, designated 5,000 times of bootstrapping and established the confidence interval of $95 \%$. The outcomes of the moderated mediating effect of the adverse childhood experiences are as [Table 1].

Table 2. Verification of a mediating effect moderated by the adverse childhood experiences

\begin{tabular}{|c|c|c|c|c|c|c|c|c|}
\hline \multicolumn{3}{|c|}{ Variable } & $\beta$ & SE & $t$ & $p$ & LLCI & ULCI \\
\hline \multicolumn{9}{|c|}{ Dependent variable model (Dependent variable: Smartphone addiction tendency) } \\
\hline \multicolumn{3}{|c|}{ Constant } & 1.071 & .203 & 5.274 & .000 & .672 & 1.469 \\
\hline Psychological acceptance & $\rightarrow$ & \multirow{2}{*}{$\begin{array}{c}\text { Smartphone addiction } \\
\text { tendency }\end{array}$} & -.213 & .032 & -6.581 & .000 & -.276 & -.149 \\
\hline Internalizing problems & $\rightarrow$ & & .027 & .004 & 7.043 & .000 & .019 & .035 \\
\hline \multicolumn{9}{|c|}{ Parameter model (Dependent variable: Internalizing problems) } \\
\hline \multicolumn{3}{|c|}{ Constant } & 52.312 & .282 & 185.165 & .000 & 51.757 & 52.867 \\
\hline Psychological acceptance & $\rightarrow$ & Internalizing problems & -5.086 & .293 & -17.310 & .000 & -5.663 & -4.509 \\
\hline $\begin{array}{l}\text { Adverse childhood } \\
\text { experiences }\end{array}$ & $\rightarrow$ & Internalizing problems & .192 & .092 & 2.076 & .038 & .010 & .375 \\
\hline $\begin{array}{c}\text { Psychological acceptance } \\
\times \text { Adverse childhood } \\
\text { experiences }\end{array}$ & $\rightarrow$ & Internalizing problems & -.221 & .089 & -2.477 & .013 & -.396 & -.046 \\
\hline \multirow{2}{*}{\multicolumn{3}{|c|}{ A rise in $R^{2}$ according to interaction }} & \multicolumn{2}{|c|}{$R^{2}$} & \multicolumn{2}{|c|}{$\mathrm{F}$} & \multicolumn{2}{|c|}{$p$} \\
\hline & & & \multicolumn{2}{|c|}{.007} & \multicolumn{2}{|c|}{6.136} & \multicolumn{2}{|c|}{.013} \\
\hline
\end{tabular}

First of all, a mediating effect of the internalizing problems was examined in a relationship between the psychological acceptance and the smartphone addiction tendency based on the dependent variable model. The psychological acceptance had a significant negative impact on smartphone addiction tendency $(\beta=-.213, \mathrm{p}<.05)$. The internalizing problems appeared to have a significant positive impact on smartphone addiction tendency $(\beta=.027, p<.05)$. This can be known to have a statistically significant mediating effect as the psychological acceptance was indicated to have significant negative influence upon internalizing problems in the 
parameter model $(\beta=-5.086, \mathrm{p}<.05)$. In the parameter model that established the internalizing problems as a dependent variable, the interaction term between the psychological acceptance and the adverse childhood experiences was indicated to have significant negative influence upon internalizing problems $(\beta=-.221, \mathrm{p}<.05)$, thereby being able to be known to have a statistically significant moderating effect. That is to say, a rise in psychological acceptance leads to a decline in internalizing problems. This comes to diminish smartphone addiction tendency. This mediation path comes to be altered depending on adverse childhood experience level. As a procedure for specifically confirming the aspect that the mediating effect of the internalizing problems is changed depending on the adverse childhood experience level as a moderating variable, the significance was validated in the mediating effect moderated by the average value (2.045) and $\pm 1 \mathrm{SD}$ (3.094) value of a moderating variable. As a result, the conditional effect of psychological acceptance according to adverse childhood experience level was shown the more size of the moderated mediating indirect effect in the higher adverse childhood experience level (-1SD: -5.820 , Mean: $-5.134,+1 \mathrm{SD}:-4.578)$ and appeared to be statistically meaningful because of being not included 0 at the confidence interval of $95 \%$ (lower bound value, upper bound value) in both the average value and \pm 1 SD level. In other words, the influence of psychological acceptance upon smartphone addiction tendency mediated by internalizing problems is meant to get weaker in the higher adverse childhood experience level. And then the statistical significance of the moderated mediating effect can be decided by identifying whether the confidence interval is covering 0 or not through applying the formal test in the Index of Moderated Mediation. Consequently, the mediation index moderated by adverse childhood experiences was -.006 and was inspected the moderated mediating effect because of being not contained 0 in the lower bound value and the upper bound value $(-.012,-.002)$ at the confidence interval of $95 \%$.

\section{Discussion}

The discussion according to the results of this study are as follows. The adverse childhood experiences appeared to have a significant moderated mediating effect in the process that the undergraduates' psychological acceptance has influence upon smartphone addiction tendency after undergoing the internalizing problems. In other words, this can be known that the higher psychological acceptance leads to the lower smartphone addiction tendency mediated by internalizing problems, but that the higher adverse childhood experience level at this time leads to possibly reducing the negative (-) influence of psychological acceptance upon smartphone addiction tendency mediated by internalizing problems. This research result is in line with the finding in Lee [17]'s research targeting university students who experienced the childhood attachment trauma as saying that the psychological acceptance has significant influence upon negative emotion like anxiety and depression, and with the finding by Choi and Kim [18] as saying that the negative parenting experiences in childhood may trigger undergraduates' smartphone addiction.

According to Erikson's psychosocial developmental stages, the university students correspond to the early adult period, thereby being at the stage of proceeding with necessarily building a sense of closeness. But the childhood negative interpersonal experience comes to bring about a variety of psychosocial maladjustment such as depression, loneliness and emotional isolation, and may be further shown the tendency of relying on surreal world dubbed online in smartphone not in reality [19]. This leads to the more rise in the internalizing problems like social withdrawal, depression or anxiety with getting higher in tendency of trying to suppress and escape the moment rather than psychologically accepting own difficulty or 
problems. The negative feelings of having failed to be accepted lead to making it a state difficult to recognize on the adaptive aspect and the effective coping strategies, resulting in coming to use smartphone as an escape or a refuge and in being apt to use smartphone addictively such as arousing the tolerance, the loss of control, and the confusion with reality due to a gradual mount in usage time for smartphone.

Accordingly, a program or activity is important for reducing the internalizing problems in the aspect for preventing smartphone addiction in undergraduates. However, there is a need to examine together about not only the individual aspect but also the family environment aspect in relation to smartphone addiction by considering what a difference is shown in the influence among variables depending on the adverse childhood experiences. Moreover, there is a difference in the influence depending on the adverse childhood experiences. Yet the psychological acceptance appears to be a factor of decreasing smartphone addiction tendency. Thus, there is a need to develop and apply a program available for making it capable of coping well with a situation by using the energy of having been consumed for avoidance in a place of experiencing other productive activity or positive emotion through realizing and accepting own cognitive and emotional parts literally with using a psychological coping strategy called acceptance instead of evading the experience.

\section{Conclusion}

Through this study, it was found that the mediating effect moderated by adverse childhood experiences was verified in the influence of university students' psychological acceptance upon smartphone addiction tendency mediated by internalizing problems. Therefore, it can be found that the smartphone addiction tendency needs to be reduced as a way to reduce the internalizing problems and adverse childhood experiences of university students. The significances of this study are as follows. First, this study was examined the psychological acceptance and the internalizing problems as the variables of affecting university students' smartphone addiction tendency. There is a significance in a regard of being available for suggesting a specific program, which can diminish smartphone addiction tendency, through inquiring into the influence upon smartphone addiction tendency in the cognitive characteristic dubbed psychological acceptance and the emotional feature called internalizing problems. Second, this study was checked the mediating effect moderated by adverse childhood experiences, thereby having confirmed that the mediating effect of internalizing problems can be regulated depending on the adverse childhood experience level in the influence of university students' psychological acceptance upon smartphone addiction tendency. This is suggesting the necessity of supporting the parent education so that not only an individual but also a family can attentively prevent the adverse childhood experiences in order to decrease undergraduates' smartphone addiction tendency.

Despite this significance, the following are the limitations of this study and the suggestions for a future research. First, there is difficulty in generalizing the findings in that this study was carried out the research analysis targeting undergraduates at universities where are located in Daegu and Gyeongbuk. Accordingly, a further research needs to be progressed a generalized study available for reducing university students' smartphone addiction tendency by conducting a survey targeting undergraduates all over the country. Second, this study was verified causality among variables of affecting smartphone addiction tendency. However, in light of the finding [20] as saying that the smartphone addiction tendency is shown the higher average score in women than in men, a follow-up research needs to inquire into a difference in verification of causality depending on gender. 


\section{Acknowledgement}

This work was supported by the National Research Foundation of Korea (2019S1A5A2A0 3046211)

\section{References}

[1] https://news.kbs.co.kr/news/view.do?ncd=4135732,Feb11(2019)

[2] D. J. Kuss and M. D. Griffiths, "Online social networking and addiction: A review of the psychological literature,” International Journal of Environmental Research and Public Health, (2011), vol.8, no9, pp.35283552

[3] S. C. Hayes, K. D. Strosahl and K. G. Wilson, "Acceptance and commitment therapy: The process and practice of mindful change. New York, Guilford Press, (2011)

[4] A. Masuda, S. C. Hayes, L. Fletcher, P. J. Seignourel, K. Bunting, S. A. Herbst, M. P. Twohig, and J. Lillis, "Impact of acceptance and commitment therapy versus education on stigma toward people with psychological disorders. Behavior research and therapy," (2007), vol.45, no.11, pp.2764-2772

[5] E. Y. Koh and E. H. Kim, "The impacts of anxiety and depression on smartphone addiction: Focusing on the moderating effect of gender," Journal of Digital Convergence, (2017), vol.15, no.5, pp.419-429

[6] J. G. Allen, "Mentalizing in the development and treatment of attachment trauma," London, UK, Karnac Books, (2013)

[7] Y. O. Lee and D. H. Ahn, "The effect of art therapy on traumatized self-system, depression, and interpersonal problems of a woman with interpersonal trauma experience,” Korean Journal of Art Therapy, (2017), vol.24, no.4, pp.1057-1081

[8] I. K. Kang and C. K. Kim, "The structural relationships between adolescents' mobile phone dependency and their related variables according to level of abuse experience: Focused on peer attachment," self-regulated learning ability, internalizing and externalizing problem behaviors, Journal of Future Oriented Youth Society, (2018), vol.15, no.2, pp.33-57

[9] J. H. Heo, M. S. Chio, and H. J. Jin, "Study on the reliability and validity of Korean translated acceptanceaction questionnaire-2," Korean Journal of Counseling and Psychotherapy, (2009), Vol.21, No.4, pp.861-878

[10] S. C. Hayes, "Acceptance and commitment therapy, relational frame theory, and the third wave of behavioral and cognitive therapies. Behavior Therapy," (2004), vol.35, no.4, pp.639-665

[11] P. T. Costa Jr. and R. R. McCrae, "Four ways five factors are basic. Personality and individual differences," (1992), vol.13, No.6, pp.653-665

[12] H. N. Ahn and C. K. Ahn, "NEO-II personality assessment interpretation guidelines," Seoul, Hakjisa, (2015)

[13] J. H. Lee, J. M. Lim, H. B. Son, H. W. Kwak, and M. S. Chang, "Development and validation of a smartphone addiction scale based on behavioral addiction criteria," Korean Journal of Counseling and Psychotherapy, (2016), vol.28, no.2, pp.425-443

[14] M. A. Straus, "Measuring intrafamily conflict and violence: The conflict tactics (CT) scales," Journal of Marriage and the Family, (1979), vol.41, no.1, pp.75-88

[15] D. P. Bernstein, L. Fink, L. Handelsman, J. Foote, M. Lovejoy, K. Wenzel, E. Saparem, and J. Ruggiero, "Initial reliability and validity of a new retrospective measure of child abuse and neglect," The American Journal of Psychiatry, (1994), vol.151, no.8, pp.1132-1136

[16] R. B. Kline, "Principles and practice of structural equation modeling (4th ed)," New York, The Guilford Press, (2015)

[17] H. S. Lee, "The mediating effects of emotional clarity and psychological acceptance on the relationship between mentalization and depression and anxiety in adulthood: For adults with emotional trauma in childhood," master's dissertation, The Catholic University, Korean, (2019) 
[18] H. I. Choi and J. H. Kim, "A study on mediation effect of depression on the relations between experience of child neglect and smartphone addiction," Journal of the Korean Society of Child Welfare, (2013), vol.44, pp.127-153

[19] E. H. Erikson, “Childhood and society,” New York, WW Norton and Company, (1963)

[20] S. J. Lee, "An analysis on the mental health of adolescent addicted to smartphone," Korena Journal of Youth Welfare, (2018), vol.20, no.3, pp.47-67 
The Influence of Psychological Acceptance and Internalizing in University Students upon Smartphone Addiction: The Moderated Mediating effect of Adverse Childhood Experiences

This page is empty by intention. 